\title{
On the Cauchy difference of functions bounded modulo $\mathbb{Z}$ on "large" sets
}

\author{
ELIZA JABŁOŃSKA(D)
}

\begin{abstract}
Let $X$ be a real Polish vector space, $D \subset X$ be a universally Baire non-Haarmeager set, and let $f: X \rightarrow \mathbb{R}$ satisfy $f(x+y)-f(x)-f(y) \in \mathbb{Z}$ for all $x, y \in X$ and $f(D) \subset\left(-\frac{1}{3}, \frac{1}{3}\right)+\mathbb{Z}$. We prove that there exists a unique linear functional $L: X \rightarrow \mathbb{R}$ with $f(x)-L(x) \in \mathbb{Z}$ for $x \in X$ and next we apply it to functionals which are orthogonally additive modulo $\mathbb{Z}$. These new results are topological counterparts to some results of Brzdęk (Results Math 30:25-38, 1996) and also generalize (to some extent) his results.
\end{abstract}

Mathematics Subject Classification. 39B52, 39B55, 54B30, 54E52.

Keywords. Cauchy difference, Real Polish vector space, Universal Baire property, Haarmeager set, Christensen zero set.

\section{Introduction}

Let $X$ be a real vector space and let $f: X \rightarrow \mathbb{R}$ satisfy the following condition:

$$
f(x+y)-f(x)-f(y) \in \mathbb{Z} \text { for } x, y \in X,
$$

where $\mathbb{Z}$ denotes the set of all integers.

The Cauchy difference has been studied by lots of authors in connection with the stability problem of the Cauchy equation. Thanks to the van der Corput Theorem (see [15, p. 64]) and its extension due to Baron and Volkmann $[4$, Theorem 1] the following result is proved.

Theorem 1. [4, Corollary 2] If $\gamma \in\left(0, \frac{1}{4}\right), f: X \rightarrow \mathbb{R}$ satisfies (1) and $D \subset X$ is a set such that

$$
\operatorname{int}(D-D) \neq \emptyset
$$

and

$$
f(D) \subset(-\gamma, \gamma)+\mathbb{Z},
$$


then there exists a continuous linear functional $L: X \rightarrow \mathbb{R}$ satisfying

$$
f(x)-L(x) \in \mathbb{Z} \text { for } x \in X .
$$

By the Steinhaus-Pettis Theorem (see [13,14], and also [12, Theorems 2.9.1 and 3.7.1]) non-meager sets with the Baire property in a real topological vector space as well as sets of positive Lebesgue measure in $\mathbb{R}^{n}$ are natural examples of sets satisfying (2).

Other examples of such sets in an abelian Polish group are Christensen measurable but not Christensen zero sets and universally Baire non-Haarmeager sets.

The definition of a Christensen zero set in an abelian Polish group $X$ was introduced in 1972 by Christensen [6] and is based on the notion of a universally measurable set, i.e. a set which is measurable with respect to the completion of the Borel field with respect to the family of all probability measures on $X$.

Definition 1. A subset $A$ of an abelian Polish group $X$ is called a Christensen zero set provided there are a universally measurable set $B \subset X$ with $A \subset B$ and a Borel probability measure $\mu$ on $X$ such that $\mu(x+B)=0$ for all $x \in X$.

Over forty years later Darji [7] introduced another family of "small" sets in an abelian Polish group $X$, called by him Haar-meager, using the $\sigma$-field of Borel sets. But it is easy to check that all results from [7] hold if we replace the $\sigma$-field of Borel sets by the larger $\sigma$-field of all universally Baire sets.

Let us recall that a set $D \subset X$ is called universally Baire (see e.g. $[1,8]$ ) if for every compact Hausdorff metric space $K$ and every continuous function $f: K \rightarrow X$ the set $f^{-1}(D)$ has the Baire property in $K$.

Definition 2. A subset $A$ of an abelian Polish group $X$ is called Haar-meager if there are a universally Baire set $B \subset X$ with $A \subset B$, a compact metric space $K$ and a continuous function $f: K \rightarrow X$ such that $f^{-1}(B+x)$ is meager in $K$ for every $x \in X$.

In a locally compact abelian Polish group Definitions 1 and 2 coincide with definitions of sets of Haar measure zero and meager sets, respectively. That is why we can say that the notion of a Haar-meager set is a topological analogue to the notion of a Christensen zero set in an abelian Polish group (some similarities can be found e.g. in $[2,11]$ ).

In view of Christensen's result [6, Theorem 2] condition (2) holds for every universally measurable set which is not a Christensen zero set, and consequently, following Fischer and Słodkowski [9] (who introduced the notion of a Christensen measurable set as a set which is a union of a universally measurable set and a Christensen zero one), (2) also holds for every Christensen 
measurable set which is not a Christensen zero set. It turns out that universally Baire non-Haar-meager sets satisfy (2), too (see [10, Theorem 2] ${ }^{1}$ ). Consequently, from Theorem 1 we get the following result.

Corollary 2. Assume that one of the following conditions is valid:

(i) $X$ is a real topological vector space and $D \subset X$ is a non-meager set with the Baire property;

(ii) $X$ is a real Polish vector space and $D \subset X$ is a Christensen measurable set which is not a Christensen zero set ${ }^{2}$;

(iii) $X$ is a real Polish vector space and $D \subset X$ is a universally Baire nonHaar-meager set.

If $f: X \rightarrow \mathbb{R}$ satisfies (1) and condition (3) holds with $\gamma \in\left(0, \frac{1}{4}\right)$, then there exists a unique ${ }^{3}$ continuous linear functional $L: X \rightarrow \mathbb{R}$ satisfying (4).

It turns out that in Theorem $1 \gamma$ can not be increased to $\frac{1}{4}$. More precisely, there exist a discontinuous additive function $f: \mathbb{R} \rightarrow \mathbb{R}$ and a set $D \subset \mathbb{R}$ such that (2) and (3) with $\gamma=\frac{1}{4}$ hold (see [4, Remark 1]).

Nonetheless, Brzdȩk [5] directly proved a stronger result than Corollary 2(i),(ii) (where (3) holds for $\gamma=\frac{1}{3}$ ).

Theorem 3. [5, Theorem 2, Corollary 3] Assume that one of the following conditions is valid:

(i) $X$ is a real Polish vector space and $D \subset X$ is a Christensen measurable set which is not a Christensen zero set;

(ii) $X$ is a real topological vector space and $D \subset X$ is a non-meager set with the Baire property.

If $f: X \rightarrow \mathbb{R}$ satisfies (1) and condition (3) holds with $\gamma=\frac{1}{3}$, then there exists a unique linear functional $L: X \rightarrow \mathbb{R}$ satisfying (4).

The following natural question arises.

Question 1. What about a topological counterpart to Theorem 3(i)? More precisely: can Corollary 2(iii) be generalized to the case when (3) holds for $\gamma=\frac{1}{3}$ ?

The aim of the paper is to answer these questions. We prove Theorem 3 assuming the very condition (iii) of Corollary 2. Our result also generalizes, to some extent, Theorem 3(ii).

\footnotetext{
${ }^{1}$ Actually, the mentioned result is obtained for Borel non-Haar-meager sets, but the proof also works for "large" sets in the sense of Definition 2.

${ }^{2}$ In the case $X=\mathbb{R}^{n}$ this condition means that $D$ is of positive Lebesgue measure.

${ }^{3}$ Uniqueness is a consequence of that there is at most one additive function $f: X \rightarrow \mathbb{R}$ satisfying (4) (see [5, Remark 1]).
} 


\section{Preliminaries}

In the beginning let us recall two technical lemmas proved by Brzdęk in [5].

Lemma 4. [5, Lemma 1] Let $X$ be a real vector space, $\emptyset \neq D \subset X$ and $f$ : $X \rightarrow \mathbb{R}$ be a function satisfying (1) and

$$
f(D) \subset\left(-\frac{1}{3}, \frac{1}{3}\right)+\mathbb{Z} .
$$

Then

$$
f\left(\left(\frac{1}{2} D\right) \cap D\right) \subset\left(-\frac{1}{6}, \frac{1}{6}\right)+\mathbb{Z} .
$$

Lemma 5. [5, Lemma 2] Let $X$ be a real vector space, $\emptyset \neq D \subset X$ and $f$ : $X \rightarrow \mathbb{R}$ be a function satisfying (1) and (5). Let $x_{0} \in D$ and $D_{0}:=D-x_{0}$. The following two conditions are valid:

(i) if $f\left(x_{0}\right) \in\left(-\frac{1}{6}, \frac{1}{6}\right)+\mathbb{Z}$, then

$$
f\left(-D_{0} \cap D_{0}\right) \subset\left(-\frac{1}{3}, \frac{1}{3}\right)+\mathbb{Z} ;
$$

(ii) if $f\left(x_{0}\right) \in\left(\left(-\frac{1}{3},-\frac{1}{6}\right] \cup\left[\frac{1}{6}, \frac{1}{3}\right)\right)+\mathbb{Z}$, then

$$
f\left(2\left(-D_{0} \cap D_{0}\right)\right) \subset\left(-\frac{1}{3}, \frac{1}{3}\right)+\mathbb{Z} .
$$

We also need the definition of an algebraically interior point.

Definition 3. Let $X$ be a real vector space. We say that $x \in X$ is an algebraically interior point of a set $D \subset X$, if for every $z \in X \backslash\{0\}$ there is $c>0$ such that $x+a z \in D$ for every $a \in(-c, c)$.

In [5] Brzdęk proved the following result which will be useful in the sequel.

Proposition 6. [5, Corollary 1, Remark 1] Let $X$ be a real vector space, $D \subset$ $X$ be a set possessing an algebraically interior point and $f: X \rightarrow \mathbb{R}$ be a function satisfying (1) and (3) with $\gamma \in\left(0, \frac{1}{2}\right)$. Then there exists a unique linear functional $L: X \rightarrow \mathbb{R}$ such that condition (4) holds.

\section{The main result}

Now, we are ready to prove a topological analog to Theorem 3(i).

Theorem 7. Let $X$ be a real Polish vector space and $D \subset X$ be a universally Baire non-Haar-meager set. If $f: X \rightarrow \mathbb{R}$ is a function satisfying (1) and (5), then there exists a unique linear functional $L: X \rightarrow \mathbb{R}$ fulfilling (4). 
Proof. First we prove that there exists a set $A \subset X$ such that 0 is an algebraically interior point of $A-A$ and

$$
f(A) \subset\left(-\frac{1}{6}, \frac{1}{6}\right)+\mathbb{Z} .
$$

To this end fix $x \in X \backslash\{0\}$. Choose a compact interval $I \subset \mathbb{R}$ and define $k_{x}: \mathbb{R} \rightarrow X$ by $k_{x}(t)=t x$ for $t \in \mathbb{R}$. Since $D$ is a universally Baire non-Haarmeager set, for the continuous function $f_{x}:=\left.k_{x}\right|_{I}$ mapping $I$ into $X$ there exists $y_{x} \in X$ such that the set $k_{x}^{-1}\left(D+y_{x}\right) \cap I=f_{x}^{-1}\left(D+y_{x}\right)$ is not meager and has the Baire property in $I$; consequently $T_{x}:=k_{x}^{-1}\left(D+y_{x}\right)$ is not meager and has the Baire property in $\mathbb{R}$. It means that $T_{x}=\left(U_{x} \backslash I_{x}\right) \cup J_{x}$ for some non-empty open set $U_{x} \subset \mathbb{R}$ and meager sets $I_{x}, J_{x}$. Put $S_{x}:=U_{x} \backslash I_{x} \subset T_{x}$. Clearly, $T_{x} x-y_{x} \subset D$ so, according to (5),

$$
f\left(S_{x} x-y_{x}\right) \subset f\left(T_{x} x-y_{x}\right) \subset f(D) \subset\left(-\frac{1}{3}, \frac{1}{3}\right)+\mathbb{Z} .
$$

Choose $c_{x} \in S_{x}$; hence $f\left(c_{x} x-y_{x}\right) \subset\left(-\frac{1}{3}, \frac{1}{3}\right)+\mathbb{Z}$. Then, by Lemma 5 (with $D=S_{x} x-y_{x}$ and $\left.x_{0}=c_{x} x-y_{x}\right)$, we obtain:

(i) if $f\left(c_{x} x-y_{x}\right) \in\left(-\frac{1}{6}, \frac{1}{6}\right)+\mathbb{Z}$, then

$$
f\left(\left[\left(S_{x}-c_{x}\right) \cap\left(-S_{x}+c_{x}\right)\right] x\right) \subset\left(-\frac{1}{3}, \frac{1}{3}\right)+\mathbb{Z} ;
$$

(ii) if $f\left(c_{x} x-y_{x}\right) \in\left(-\frac{1}{3},-\frac{1}{6}\right] \cup\left[\frac{1}{6}, \frac{1}{3}\right)+\mathbb{Z}$, then

$$
f\left(2\left[\left(S_{x}-c_{x}\right) \cap\left(-S_{x}+c_{x}\right)\right] x\right) \subset\left(-\frac{1}{3}, \frac{1}{3}\right)+\mathbb{Z} .
$$

Now, put

$$
W_{x}:=\left\{\begin{array}{l}
\left(S_{x}-c_{x}\right) \cap\left(-S_{x}+c_{x}\right), \quad \text { if } f\left(c_{x} x-y_{x}\right) \in\left(-\frac{1}{6}, \frac{1}{6}\right)+\mathbb{Z} ; \\
2\left[\left(S_{x}-c_{x}\right) \cap\left(-S_{x}+c_{x}\right)\right], \text { if } f\left(c_{x} x-y_{x}\right) \in\left(-\frac{1}{3},-\frac{1}{6}\right] \cup\left[\frac{1}{6}, \frac{1}{3}\right)+\mathbb{Z} .
\end{array}\right.
$$

Since

$$
V_{x}:=\left\{\begin{array}{l}
\left(U_{x}-c_{x}\right) \cap\left(-U_{x}+c_{x}\right), \quad \text { if } f\left(c_{x} x-y_{x}\right) \in\left(-\frac{1}{6}, \frac{1}{6}\right)+\mathbb{Z} ; \\
2\left[\left(U_{x}-c_{x}\right) \cap\left(-U_{x}+c_{x}\right)\right], \text { if } f\left(c_{x} x-y_{x}\right) \in\left(-\frac{1}{3},-\frac{1}{6}\right] \cup\left[\frac{1}{6}, \frac{1}{3}\right)+\mathbb{Z}
\end{array}\right.
$$

is a symmetric neighbourhood of $0, W_{x} \cap V_{x}$ is co-meager in $V_{x}$. Hence $W_{x}$ is a non-meager set with the Baire property in $\mathbb{R}$ and

$$
f\left(W_{x} x\right) \subset\left(-\frac{1}{3}, \frac{1}{3}\right)+\mathbb{Z} .
$$


Now, let $A_{x}:=W_{x} \cap\left(\frac{1}{2} W_{x}\right)$. Clearly, $A_{x} \cap\left(\frac{1}{2} V_{x}\right)$ is co-meager in $\frac{1}{2} V_{x} \subset V_{x}$. Thus $A_{x}$ is a non-meager set with the Baire property in $\mathbb{R}$ and, by the Pettis Lemma (see [13, Corollary 5]), $0 \in \operatorname{int}_{\mathbb{R}}\left(A_{x}-A_{x}\right)$. Consequently, 0 is the algebraically interior point of the set $A-A$, where $A:=\bigcup_{x \in X \backslash\{0\}} A_{x} x$.

Moreover, in view of Lemma 4 (with $D=W_{x} x$ ),

$$
f\left(A_{x} x\right) \subset\left(-\frac{1}{6}, \frac{1}{6}\right)+\mathbb{Z}
$$

and condition (6) holds, which ends the proof of the first step.

Further, setting $x=y=0$ and next $y=-x$ in (1), we get $f(0) \in \mathbb{Z}$ and hence

$$
f(x)+f(-x) \in \mathbb{Z} \text { for } x \in X .
$$

Thus

$$
f(A-A) \subset f(A)-f(A)+\mathbb{Z} \subset\left(-\frac{1}{3}, \frac{1}{3}\right)+\mathbb{Z}
$$

and, in view of Proposition 6, we obtain the thesis.

In [7] Darji proved that every Haar-meager set is meager, but the converse implication holds if and only if the abelian Polish group considered is locally compact. That is why Theorem 7 generalizes, to some extent, Theorem 3(ii).

\section{An application to functionals which are orthogonally additive modulo $\mathbb{Z}$}

Let us recall the following result of Brzdęk.

Theorem 8. [5, Theorem 3] Let $(X,\langle\cdot, \cdot\rangle)$ be a real inner product space with $\operatorname{dim} X>1$. Assume also that one of the following conditions holds:

(i) $X$ is a Polish space and $D \subset X$ is a Christensen measurable set which is not a Christensen zero set,

(ii) $D \subset X$ is a non-meager set with the Baire property.

If $f: X \rightarrow \mathbb{R}$ is a functional satisfying

$$
f(x+y)-f(x)-f(y) \in \mathbb{Z} \text { for all } x, y \in X \text { with }\langle x, y\rangle=0
$$

and

$$
f(D) \subset\left(-\frac{1}{6}, \frac{1}{6}\right)+\mathbb{Z},
$$

then there exists a unique linear functional $L: X \rightarrow \mathbb{R}$ and a unique $\alpha \in \mathbb{R}$ with

$$
f(x)-\alpha\|x\|^{2}-L(x) \in \mathbb{Z} \text { for } x \in X .
$$


Now, in exactly the same way as in the proof of Theorem 8 (see $[5$, the proof of Theorem 3]) we can derive a topological counterpart to Theorem 8(i).

Corollary 9. Let $(X,\langle\cdot, \cdot\rangle)$ be a real inner product space with $\operatorname{dim} X>1$ and $D \subset X$ be a universally Baire non-Haar-meager set. If $f: X \rightarrow \mathbb{R}$ is a functional satisfying (7) and (8), then there exists a unique linear functional $L: X \rightarrow \mathbb{R}$ and a unique $\alpha \in \mathbb{R}$ with (9).

Since each non-meager set is non-Haar-meager, Corollary 9 in some sense generalizes Theorem 8(ii). Moreover, it also refers to [3, Corollary 1] due to Baron and Forti.

Open Access. This article is licensed under a Creative Commons Attribution 4.0 International License, which permits use, sharing, adaptation, distribution and reproduction in any medium or format, as long as you give appropriate credit to the original author(s) and the source, provide a link to the Creative Commons licence, and indicate if changes were made. The images or other third party material in this article are included in the article's Creative Commons licence, unless indicated otherwise in a credit line to the material. If material is not included in the article's Creative Commons licence and your intended use is not permitted by statutory regulation or exceeds the permitted use, you will need to obtain permission directly from the copyright holder. To view a copy of this licence, visit http:// creativecommons.org/licenses/by/4.0/.

Publisher's Note Springer Nature remains neutral with regard to jurisdictional claims in published maps and institutional affiliations.

\section{References}

[1] Bagaria, J., Castells, N., Larson, P.: An $\Omega$-logic primer. In: Set Theory, pp. 1-28, Trends Math. Birkhäuser, Basel (2006)

[2] Banakh, T., Głąb, S., Jabłońska, E., Swaczyna, J.: Haar-I sets: looking at small sets in Polish groups through compact glasses, arXiv:1803.06712 [math.GN]

[3] Baron, K., Forti, G.L.: Othogonality and additivity modulo $\mathbb{Z}$. Results Math. 26, 205210 (1994)

[4] Baron, K., Volkmann, P.: On a theorem of van der Corput. Abh. Math. Sem. Univ. Hamburg 61, 189-195 (1991)

[5] Brzdȩk, J.: On functionals which are orthogonally additive modulo $\mathbb{Z}$. Results Math. 30, 25-38 (1996)

[6] Christensen, J.P.R.: On sets of Haar measure zero in abelian Polish groups. Israel J. Math. 13, 255-260 (1972)

[7] Darji, U.B.: On Haar meager sets. Topol. Appl. 160, 2396-2400 (2013)

[8] Feng, Q., Magidor, M., Woodin, H.: Universally Baire sets of reals. In: Judah, H., Just, W., Woodin, H. (eds.) Set theory of the continuum, pp. 203-242. Math. Sci. Res. Inst. Publ. 26, Springer, New York (1992)

[9] Fischer, P., Słodkowski, Z.: Christensen zero sets and measurable convex functions. Proc. Am. Math. Soc. 79, 449-453 (1980)

[10] Jabłońska, E.: Some analogies between Haar meager sets and Haar null sets in abelian Polish groups. J. Math. Anal. Appl. 421, 1479-1486 (2015) 
[11] Jabłońska, E.: Remarks on analogies between Haar meager sets and Haar null sets. In: Brzdęk, J., Ciepliński, K., Rassias, Th.M. (eds.) Developments in Functional Equations and Related Topics, pp. 149-159. Springer Optimization and its Applications 124, Springer (2017)

[12] Kuczma, M.: An introduction to the theory of functional equations and inequalities. Cauchy's Equation and Jensen's Inequality. 2nd edn, Gilányi, A. (ed.), Birkhäuser, Basel (2009)

[13] Pettis, B.J.: Remarks on a theorem of E. J. McShane. Proc. Am. Math. Soc. 2, 166-171 (1951)

[14] Steinhaus, H.: Sur les distances des points dans les ensembles de mesure positive. Fund. Math. 1, 93-104 (1920)

[15] van der Corput, J.G.: Goniometrische functies gekarakteriseerd door een functionaalbetrekking. Euclides 17, 55-75 (1940)

Eliza Jabłońska

Institute of Mathematics

Pedagogical University of Krakow

Podchorążych 2

30-084 Kraków

Poland

e-mail: eliza.jablonska@up.krakow.pl

Received: February 11, 2020

Revised: July 22, 2020 